\section{Channel Estimation for OFDMA Uplink: a Hybrid of Linear and BEM Interpolation Approach}

Yi Ma, Member, IEEE, and Rahim Tafazolli

\begin{abstract}
We consider the channel estimation for the subband orthogonal frequency-division multiple-access (OFDMA) uplink. The channel for each subband is modeled by combing the linear and Fourier basis-expansion functions. Based on this mode, a two-dimensional interpolation approach is proposed for the channel estimation, which is carefully investigated in terms of estimation error and pilot placement.
\end{abstract}

Index Terms-Channel estimation, Doppler shifts, interpolation, orthogonal frequency-division multiple access (OFDMA), time varying, uplink.

\section{INTRODUCTION}

Orthogonal frequency-division multiple-access (OFDMA) is being considered to be the multiple access scheme for the uplink of future wireless systems, e.g., WiMax or 4G in Europe. One of typical structures is the subband OFDMA, which divides all available subcarriers into a number of subbands. Each user is allowed to use one available subband for the data transmission. Pilot symbols are employed for the estimation of channel state information (CSI) within the subband. We can see that the CSI here is the partial channel information of each user channel. The channel estimation algorithms used for obtaining the channel impulse response (CIR) (e.g., [1]-[3]) may not be applicable in this scenario. Practically, placement of pilot symbols obeys the criterion: channel fades between any two adjacent pilot symbols (either in time or frequency direction) can be regarded to be linear. The polynomial-based interpolation (e.g., [4]) can be employed for the channel estimation. However, the polynomial-based interpolation is sensitive to the pilot spacing. Its denoising capability is restricted due to avoiding considerable modeling mismatch. It is noted by the reviewer that iterative approaches (e.g., [9] and [10]) have recently received great interest in the channel estimation. In this paper, we only focus on the model-based interpolation. The result may be used for the initial channel estimate of the iterative approaches.

Giannakis and Tepedelenlioglu mentioned in [5] that the time-frequency-selective channel can be modeled as Fourier basis expansion. Thereafter, this model was intensively investigated and applied in block transmission, channel estimation and equalization (e.g., [6]-[8]). In an OFDM system, the channel fades on the $n$ block of the $m$ th subcarrier is given by (see [8])

$\tilde{h}(n, m)=\sum_{q=-Q}^{Q} \underbrace{\sum_{\ell=0}^{L_{u}} \gamma_{q}(\ell) \exp \left(\frac{-j 2 \pi m \ell}{M}\right)}_{=\tilde{\gamma}_{q}(m)} \exp \left(j \frac{2 \pi n \bar{f}_{\max } q}{Q}\right)$

where $\gamma_{q}(\ell)$ stands for the fading coefficient of the $q$ th path in the $\ell$ th channel tap, $\bar{f}_{\max }$ for the maximum Doppler shift normalized by the

Manuscript received November 11, 2005; revised February 27, 2006. This work was supported by European Commission under IST 4More project, No. IST-2002-507039. The associate editor coordinating the review of this paper and approving it for publication was Prof. Philippe Loubaton.

The authors are with the Center for Communication Systems Research, University of Surrey, Surrey GU2 7XH, U.K. (e-mail: y.ma@surrey.ac.uk; r.tafazolli@surrey.ac.uk).

Digital Object Identifier 10.1109/TSP.2006.887559 block duration, $Q$ for the number of paths, ${ }^{1} L_{u}$ for the upper bound of channel order, and $M$ for the number of discrete Fourier transform (DFT) grids.

In this paper, we combine linear and basis expansion model (BEM) to describe the time-frequency selectivity within each subband. This two-dimensional model offers hybrid of linear and BEM interpolation (HLBI) for the channel estimation. The proposed approach is investigated both in single-transmit-antenna and multiple-transmitantenna scenarios. The channel estimation performance, together with pilot placement, is carefully studied. We compare the HLBI approach with the time-frequency linear interpolation (TFLI) approach and find that the former one shows better performance, particularly in the low signal-to-noise ratio (SNR), larger Doppler, or multiple-transmitantenna scenario.

\section{OFDMA UPLINK AND CHANNEL MODEL}

We consider cyclic prefix (CP) OFDM to be the air interface, where $\mathrm{CP}$ is used to mitigate the interblock interference and introduce the circulant property (see [12] for detail). The multiuser channel is assumed to be invariant within one-block (or -symbol) duration. The symbol timing mismatch is assumed to be smaller than the $\mathrm{CP}$ duration. In this scenario, users do not interfere each other in the frequency domain.

We assume that the desired subband consists of $(K+1)$ subcarriers. The channel fades within this subband can be formulated as

$$
\tilde{h}(n, m)=a_{1}(n) m+a_{2}(n), \quad K_{o} \leqslant m \leqslant K_{o}+K
$$

where $a_{1}$ and $a_{2}$ stand for TV coefficients, and $K_{o}$ for the index of the start subcarrier within the subband. Without loss of generality, we let $K_{o}=0$ and the received block index from 0 to $N-1$. Equations (1) and (2) lead to

$$
\begin{gathered}
a_{1}(n) m+a_{2}(n)=\sum_{q=-Q}^{Q} \tilde{\gamma}_{q}(m) \exp \left(j \frac{2 \pi n \bar{f}_{\max } q}{Q}\right), \\
0 \leqslant n \leqslant N-1, \quad 0 \leqslant m \leqslant K .
\end{gathered}
$$

We construct a $(K+1) \times N$ matrix $\tilde{\boldsymbol{H}}$ with the $(k, n)$ th entry of $[\tilde{\boldsymbol{H}}]_{k, n}=\tilde{h}(n, k), \mathrm{a}(K+1) \times(2 Q+1)$ matrix $\tilde{\Gamma}$ with the $(k, q)$ th entry of $[\tilde{\boldsymbol{\Gamma}}]_{k, q}=\tilde{\gamma}_{q-Q}(k)$, a $(2 Q+1) \times N$ matrix $\boldsymbol{U}$ with the $(q, n)$ th entry of $[\boldsymbol{U}]_{q, n}=U_{q-Q}(n)$ for

$$
U_{q}(n)=\exp \left(j \frac{2 \pi n \bar{f}_{\max } q}{Q}\right), \quad-Q \leqslant q \leqslant Q .
$$

as well as the following two matrices:

$$
\begin{aligned}
\mathcal{L} & =\left[\begin{array}{llll}
1, & 1, & \ldots, & 1 \\
0, & 1, & \ldots, & K
\end{array}\right]^{T} \\
\boldsymbol{A} & =\left[\begin{array}{llll}
a_{1}(0), & a_{1}(1), & \ldots, & a_{1}(N-1) \\
a_{2}(0), & a_{2}(1), & \ldots, & a_{2}(N-1)
\end{array}\right]
\end{aligned}
$$

where the superscript ${ }^{T}$ stands for transpose. Then, (2) and (3) can be re-expressed as the following matrix forms, respectively:

$$
\begin{aligned}
\tilde{\boldsymbol{H}} & =\mathcal{L} \boldsymbol{A} \\
\mathcal{L} \boldsymbol{A} & =\tilde{\Gamma} \boldsymbol{U} .
\end{aligned}
$$

Since $\mathcal{L}$ has the full column rank, we can obtain

$$
\tilde{\boldsymbol{H}}=\mathcal{L} \underbrace{\mathcal{L}}_{=\overline{\boldsymbol{A}}} \underbrace{\dagger} \tilde{\boldsymbol{\Gamma}} U
$$

where the superscript ${ }^{\dagger}$ denotes pseudo inverse. Now, the problem becomes the estimation of the $2 \times(2 Q+1)$ matrix $\overline{\boldsymbol{A}}$.

${ }^{1}$ It was pointed out in [5] that $Q$ should be sufficiently large (e.g., $Q=4$ or $5)$ to simulate mobile communication channel. 


\section{Channel Estimation And Pilot Placement}

\section{A. Channel Estimation}

We employ pilot symbols to identify $\overline{\boldsymbol{A}}$ in the noisy scenario. To guarantee the channel identifiability, the number of pilot symbols should not be smaller than $4 Q+2$. We use a $(K+1) \times 1$ vector $\boldsymbol{p}(n)$ to express the pilot information at the $n$th block within the desired subband, i.e., the nonzero elements in $\boldsymbol{p}(n)$ denote pilot symbols in the frequency domain, and the zero element means no pilot is placed here. Let $\tilde{\boldsymbol{h}}(n)$ and $\boldsymbol{u}(n)$ be the $n$th column vector of $\tilde{\boldsymbol{H}}$ and $\boldsymbol{U}$, respectively. The received pilot information can be expressed by

$$
\begin{aligned}
\boldsymbol{r}(n) & =\mathbf{D}(\boldsymbol{p}(n)) \tilde{\boldsymbol{h}}(n)+\boldsymbol{v}(n) \\
& =\mathbf{D}(\boldsymbol{p}(n)) \mathcal{L} \overline{\boldsymbol{A}} \boldsymbol{u}(n)+\boldsymbol{v}(n)
\end{aligned}
$$

where $\mathbf{D}(\boldsymbol{a})$ denotes a diagonal matrix with the column vector $\boldsymbol{a}$ in its diagonal, $\boldsymbol{v}(n)$ denotes additive white Gaussian noise with zero-mean and variance of $\sigma_{v}^{2}$. Our channel estimation algorithm is to minimize the following function

$$
\xi=\sum_{n=0}^{N-1}\|\boldsymbol{r}(n)-\mathbf{D}(\boldsymbol{p}(n)) \hat{\mathcal{L}} \hat{\boldsymbol{A}} \boldsymbol{u}(n)\|^{2},
$$

where $\|\cdot\|$ stands for Frobenius norm, and $\hat{\bar{A}}$ for the estimate of $\bar{A}$. It is easy to see that $\xi$ achieves its minimum when $\partial \xi / \partial \hat{\bar{A}}=0$, which is followed by

$$
\begin{aligned}
& \sum_{n=0}^{N-1} \mathcal{L}^{H} \mathbf{D}^{*}(\boldsymbol{p}(n)) \boldsymbol{r}(n) \boldsymbol{u}^{H}(n)=\sum_{n=0}^{N-1} \mathcal{L}^{H} \underbrace{\mathbf{D}(\boldsymbol{p}(n)) \mathbf{D}^{*}(\boldsymbol{p}(n))}_{=\mathbf{D}(\overline{\boldsymbol{p}}(n))} \\
& \times \hat{\mathcal{L}} \hat{\bar{A}} \boldsymbol{u}(n) \boldsymbol{u}^{H}(n)
\end{aligned}
$$

where the superscript ${ }^{H}$ stands for Hermitian. We assume that pilot symbols have the identical amplitude denoted by $\sigma_{p}$. The pilot placement complies with the following criterion ${ }^{2}$ :

$$
\overline{\boldsymbol{p}}(n)=\overline{\boldsymbol{p}}, \quad n \in \mathcal{N}_{1} ; \quad \overline{\boldsymbol{p}}(n)=\mathbf{o}, \quad n \in \mathcal{N}_{2}
$$

where $\mathcal{N}_{1} \cap \mathcal{N}_{2}=0$ and $\mathcal{N}_{1} \cup \mathcal{N}_{2}=\{0,1, \ldots, N-1\}$. Then, (12) can be rewritten into

$$
\sum_{n \in \mathcal{N}_{1}} \mathcal{L}^{H} \mathbf{D}^{*}(\boldsymbol{p}(n)) \boldsymbol{r}(n) \boldsymbol{u}^{H}(n)=\underbrace{\mathcal{L}^{H} \mathbf{D}(\overline{\boldsymbol{p}}) \mathcal{L}}_{=\overline{\mathcal{L}}} \hat{\overline{\boldsymbol{A}}} \underbrace{\sum_{n \in \mathcal{N}_{1}} \boldsymbol{u}(n) \boldsymbol{u}^{H}(n)}_{=\mathcal{U}} .
$$

We expect that the square matrices $\overline{\mathcal{L}}$ and $\mathcal{U}$ have the full rank so that $\hat{\overline{\boldsymbol{A}}}$ can be obtained via

$$
\hat{\overline{\boldsymbol{A}}}=\overline{\mathcal{L}}^{-1} \sum_{n \in \mathcal{N}_{1}} \mathcal{L}^{H} \mathbf{D}^{*}(\boldsymbol{p}(n)) \boldsymbol{r}(n) \boldsymbol{u}^{H}(n) \boldsymbol{U}^{-1} .
$$

Then, $\hat{\tilde{\boldsymbol{H}}}$ can be estimated by applying $\hat{\overline{\boldsymbol{A}}}$ in (8).

\section{B. Pilot Placement and Performance Analysis}

The first objective on pilot placement is to guarantee the channel identifiability. Therefore, $\overline{\boldsymbol{p}}$ should have at least two nonzero elements, and $\mathcal{N}_{1}$ should have at least $2 Q+1$ elements. Then, we consider the pilot placement by minimizing the mean-square channel estimation error (MSCEE).

We plug (10) into (15) and obtain

$$
\hat{\overline{\boldsymbol{A}}}=\overline{\boldsymbol{A}}+\underbrace{\overline{\mathcal{L}}^{-1} \sum_{n \in \mathcal{N}_{1}} \mathcal{L}^{H} \mathbf{D}^{*}(\boldsymbol{p}(n)) \boldsymbol{v}(n) \boldsymbol{u}^{H}(n) \mathcal{U}^{-1}}_{=\boldsymbol{E}} .
$$

Here, $\boldsymbol{E}$ is the estimation error with zero mean. Then, the MSCEE is given by

$$
\begin{aligned}
\mathcal{E}\|\boldsymbol{E}\|^{2}= & \operatorname{Tr}\left\{\overline{\mathcal{L}}^{-1} \mathcal{L}^{H} \sum_{n \in \mathcal{N}_{1}} \mathbf{D}^{*}(\boldsymbol{p}(n)) \mathcal{E}\left\{\boldsymbol{v}(n) \alpha(n) \boldsymbol{v}^{H}(n)\right\}\right. \\
& \left.\times \mathbf{D}(\boldsymbol{p}(n)) \mathcal{L}\left(\overline{\mathcal{L}}^{-1}\right)^{H}\right\} \\
= & \sigma_{v}^{2} \sum_{n \in \mathcal{N}_{1}} \alpha(n) \zeta
\end{aligned}
$$

where $\mathcal{E}$ stands for expectation, $\operatorname{Tr}\{\cdot\}$ for trace, and

$$
\begin{aligned}
\alpha(n) & =\boldsymbol{u}^{H}(n)\left(\boldsymbol{U}^{H} \boldsymbol{U}\right)^{-1} \boldsymbol{u}(n) \\
\zeta & =\operatorname{Tr}\left\{\overline{\mathcal{L}}^{-1}\right\} .
\end{aligned}
$$

Therefore, minimizing MSCEE is equivalent to minimize $\sum_{n \in \mathcal{N}_{1}} \alpha(n)$ as well as $\zeta$.

1) We re-express (4) as

$$
U_{q}(n)=\exp \left(j \frac{2 \pi n N \bar{f}_{\max } q}{N Q}\right) .
$$

Reference [6] suggested to choose $N$ and $Q$ properly so that

$$
\frac{N \bar{f}_{\max }}{Q} \approx\left\lceil\frac{N \bar{f}_{\max }}{Q}\right\rceil=1
$$

where $\lceil\cdot\rceil$ stands for integer ceiling. Then, $U$ becomes a submatrix of the $N \times N$ inverse DFT matrix. In this case, it has been shown in [1] that $\sum_{n \in \mathcal{N}_{1}} \alpha(n)$ achieves its minimum

$$
\left(\sum_{n \in \mathcal{N}_{1}} \alpha(n)\right)_{\min }=\frac{2 Q+1}{Q^{\prime}}
$$

when $\mathcal{N}_{1}=\left\{0,\left(N / Q^{\prime}\right), \ldots,\left(\left(Q^{\prime}-1\right) N\right) / Q^{\prime}\right\}$, where $Q^{\prime}$ is the number of element in $\mathcal{N}_{1}$ and fulfills $N / Q^{\prime}$ to be an integer. We also note that the computational complexity is major from the linear and BEM interpolation. Based on (21) and (22), the BEM interpolation can be implemented by employing fast Fourier transform. Therefore, the computational complexity is $o\left(2 Q^{\prime} \log Q^{\prime}+\right.$ $2 N \log N)$ for the BEM interpolation and $o(2(K+1) N)$ for the linear interpolation.

2) $\zeta$ is the error from the linear interpolation. Practically, we use two pilot symbols for linear regression. Denote $x(K>x \geqslant 0)$ and $x+d(K \geqslant d>0)$ to be the pilot placement. We can easily obtain

$$
\zeta=\frac{(x+d)^{2}+x^{2}+2}{d^{2} \sigma_{p}^{2}} .
$$

Then, $\zeta$ achieves its minimum for $x=0$ and $d=K$, i.e., $\zeta_{\min }=$ $\left(K^{2}+2\right) /\left(K^{2} \sigma_{p}^{2}\right)$. Therefore, the minimum MSCEE is given by

$$
\left(\mathcal{E}\|\boldsymbol{E}\|^{2}\right)_{\min }=\frac{\sigma_{v}^{2}(2 Q+1)\left(K^{2}+2\right)}{\sigma_{p}^{2} Q^{\prime} K^{2}} .
$$

The estimation error considered here does not take into account of the modeling mismatch. It has been addressed in [11] that the BEM using rectangular window associated with the DFT introduces spectral leakage. The energy from low-frequency Fourier coefficients leaks to the full frequency range. Slepian basis expansion is suggested to solve this problem. In this paper, however, we only consider Fourier basis expansion. 


\section{Consideration OF Multiple Transmit Antennas}

We consider a mobile terminal equipped with $I$ transmit antennas. Channel estimation is individually carried out at each receiving antenna. The pilot placement and energy for different antennas are identical. Therefore, the received pilot information is expressible as

$$
\boldsymbol{r}(n)=\sum_{i=0}^{I-1} \mathbf{D}\left(\boldsymbol{p}_{i}(n)\right) \mathcal{L} \overline{\boldsymbol{A}}_{i} \boldsymbol{u}(n)+\boldsymbol{v}(n)
$$

where the subscript $i$ stands for transmit-antenna index. The least-square channel estimation is to minimize the following function:

$$
\xi=\sum_{n=0}^{N-1}\left\|\boldsymbol{r}(n)-\sum_{i=0}^{I-1} \mathbf{D}\left(\boldsymbol{p}_{i}(n)\right) \mathcal{\mathcal { L }} \hat{\overline{\boldsymbol{A}}}_{i} \boldsymbol{u}(n)\right\|^{2} .
$$

Hence, the estimate $\hat{\overline{\boldsymbol{A}}}_{j}$ for the $j$ th transmit antenna must meet the condition $\partial \xi / \partial \hat{\overline{\boldsymbol{A}}}_{j}=0$, which is followed by

$$
\begin{aligned}
& \sum_{n=0}^{N-1} \mathcal{L}^{H} \underbrace{\mathbf{D}\left(\boldsymbol{p}_{j}(n)\right) \mathbf{D}^{*}\left(\boldsymbol{p}_{j}(n)\right)}_{=\mathbf{D}\left(\overline{\boldsymbol{p}}_{j}\right)} \mathcal{L} \hat{\overline{\boldsymbol{A}}}_{j} \boldsymbol{u}(n) \boldsymbol{u}^{H}(n) \\
& =\sum_{n=0}^{N-1} \mathcal{L}^{H} \mathbf{D}^{*}\left(\boldsymbol{p}_{j}(n)\right) \boldsymbol{r}(n) \boldsymbol{u}^{H}(n) \\
& -\mathcal{L}^{H} \sum_{n=0}^{N-1}\left(\sum_{i=0, i \neq j}^{I-1} \mathbf{D}^{*}\left(\boldsymbol{p}_{j}(n)\right) \mathbf{D}\left(\boldsymbol{p}_{i}(n)\right) \mathcal{L} \hat{\overline{\boldsymbol{A}}}_{i}\right) \quad \boldsymbol{u}(n) \boldsymbol{u}^{H}(n) .
\end{aligned}
$$

The second term at the right-hand side of (28) is the multiple-antenna interference. It is expected to be zero so that the channel estimation for multiple transmit antennas is equivalent to the case for the single transmit antenna. Then, the following condition is sufficient:

$$
\sum_{n=0}^{N-1} \mathbf{D}^{*}\left(\boldsymbol{p}_{j}(n)\right) \mathbf{D}\left(p_{i}(n)\right) \mathcal{L} \hat{\overline{\boldsymbol{A}}}_{i} \boldsymbol{u}(n) \boldsymbol{u}^{H}(n)=\mathbf{0}, \quad i \neq j .
$$

We expect to have a coefficient $g_{i j}(n)$ that makes

$$
\mathbf{D}^{*}\left(\boldsymbol{p}_{j}(n)\right) \mathbf{D}\left(\boldsymbol{p}_{i}(n)\right)=\mathbf{D}(\overline{\boldsymbol{p}}) g_{i j}(n)
$$

Using the same pilot-placement scheme as in the single-transmit-antenna case, (29) can be rewritten into

$$
\mathbf{D}(\overline{\boldsymbol{p}}) \mathcal{L} \hat{\overline{\boldsymbol{A}}}_{i} \sum_{n \in \mathcal{N}_{1}} g_{i j}(n) \boldsymbol{u}(n) \boldsymbol{u}^{H}(n)=\mathbf{0}, \quad i \neq j .
$$

Based on the principle of orthogonality, we can obtain

$$
\sum_{n \in \mathcal{N}_{1}} g_{i j}(n) \boldsymbol{u}(n) \boldsymbol{u}^{H}(n)=\mathbf{0}
$$

by employing

$$
g_{i j}(n)=\exp \left(j \frac{2 \pi n(2 Q+1)}{N}\right), \quad n \in \mathcal{N}_{1}
$$

Using the above pilot design, the channel estimation for $I$ transmitantennas needs at least $2 I(2 Q+1)$ pilot symbols. The MSCEE for each transmit antenna is the same as in the single-transmit-antenna case [see (25)].

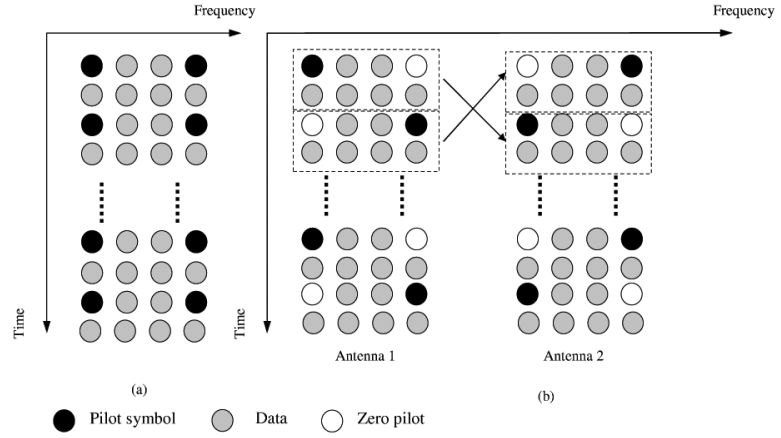

Fig. 1. Example of a subband in the OFDMA uplink: (a) single transmit antenna and (b) two transmit antennas (Alamouti scheme).

On the other hand, the pilot pattern [(30), (33)] needs the knowledge of $Q$ and $N$ at the mobile terminal, which affects the receiver flexibility. Alternatively, we can use the following pilot pattern:

$$
\mathbf{D}^{*}\left(p_{j}(n)\right) \mathbf{D}\left(p_{i}(n)\right)=\mathbf{0} .
$$

Zero pilot is employed here to avoid interantenna interference. This pilot-pattern design has received applications in practical systems (e.g., WiMax). Fig. 1(b) shows an example of (34) with two transmit antennas (Alamouti scheme [13]). In this example, (29) should be rewritten into

$$
\begin{aligned}
& \sum_{n=0}^{N-1} \mathcal{L}^{H} \underbrace{\mathbf{D}\left(\boldsymbol{p}_{j}(n)\right) \mathbf{D}^{*}\left(\boldsymbol{p}_{j}(n)\right)}_{=\mathbf{D}\left(\overline{\boldsymbol{p}}_{j}(n)\right)} \mathcal{L} \hat{\overline{\boldsymbol{A}}}_{j} \boldsymbol{u}(n) \boldsymbol{u}^{H}(n) \\
&=\sum_{n=0}^{N-1} \mathcal{L}^{H} \mathbf{D}^{*}\left(\boldsymbol{p}_{j}(n)\right) \boldsymbol{r}(n) \boldsymbol{u}^{H}(n) .
\end{aligned}
$$

Here, $\overline{\boldsymbol{p}}_{j}(n)$ is given by

$$
\overline{\boldsymbol{p}}_{j}(n)= \begin{cases}\overline{\boldsymbol{p}}_{j, 1}, & n \in \mathcal{N}_{1}, \\ \overline{\boldsymbol{p}}_{j, 2}, & n \in \mathcal{N}_{2}, \\ \mathbf{0}, & n \in \mathcal{N}_{3},\end{cases}
$$

where $\mathcal{N}_{1} \cap \mathcal{N}_{2} \cap \mathcal{N}_{3}=0, \mathcal{N}_{1} \cup \mathcal{N}_{2} \cup \mathcal{N}_{3}=\{0,1, \ldots, N-1\}$ and $\overline{\boldsymbol{p}}_{j, 1}^{T} \overline{\boldsymbol{p}}_{j, 2}=0$. Then, (35) can be further rewritten into

$$
\begin{aligned}
\sum_{\kappa=1}^{2} \mathcal{L}^{H} \mathbf{D}\left(\overline{\boldsymbol{p}}_{j, \kappa}\right) \mathcal{L} \hat{\overline{\boldsymbol{A}}}_{j} \sum_{n \in \mathcal{N}_{\kappa}^{*}} \boldsymbol{u}(n) \boldsymbol{u}^{H}(n) \\
=\sum_{\kappa=1}^{2} \sum_{n \in \mathcal{N}_{\kappa}} \mathcal{L}^{H} \mathbf{D}^{*}\left(\boldsymbol{p}_{j}(n)\right) \boldsymbol{r}(n) \boldsymbol{u}^{H}(n) .
\end{aligned}
$$

Use the following pilot placement:

$$
\begin{aligned}
& \mathcal{N}_{1}=\left\{0, \frac{N}{Q^{\prime}}, \ldots, \frac{\left(Q^{\prime}-1\right) N}{Q^{\prime}}\right\} \text { and } \\
& \mathcal{N}_{2}=\left\{0+d, \frac{N}{Q^{\prime}}+d, \ldots, \frac{\left(Q^{\prime}-1\right) N}{Q^{\prime}}+d\right\}
\end{aligned}
$$

where $d$ is an integer with $0<d<N / Q^{\prime}\left(d=N / 2 Q^{\prime}\right.$ for Alamouti scheme). We can observe

$$
\sum_{n \in \mathcal{N}_{1}} \boldsymbol{u}(n) \boldsymbol{u}^{H}(n)=\sum_{n \in \mathcal{N}_{2}} \boldsymbol{u}(n) \boldsymbol{u}^{H}(n)=\mathcal{U}
$$


under the condition $\left\lceil N \bar{f}_{\max } / Q\right\rceil=1$. In this case, (37) is expressible as

$$
\underbrace{\mathcal{L}^{H}\left(\sum_{\kappa=1}^{2} \mathbf{D}\left(\overline{\boldsymbol{p}}_{j, \kappa}\right)\right) \mathcal{L}}_{=\overline{\mathcal{L}}} \hat{\overline{\boldsymbol{A}}}_{j} \mathcal{U}=\sum_{\kappa=1}^{2} \sum_{n \in \mathcal{N}_{\kappa}} \mathcal{L}^{H} \mathbf{D}^{*}\left(\boldsymbol{p}_{j}(n)\right) \boldsymbol{r}(n) \boldsymbol{u}^{H}(n) .
$$

Therefore, $\hat{\overline{\boldsymbol{A}}}_{j}$ can be obtained via

$$
\hat{\overline{\boldsymbol{A}}}_{j}=\overline{\mathcal{L}}^{-1}\left(\sum_{\kappa=1}^{2} \sum_{n \in \mathcal{N}_{\kappa}} \mathcal{L}^{H} \mathbf{D}^{*}\left(\boldsymbol{p}_{j}(n)\right) \boldsymbol{r}(n) \boldsymbol{u}^{H}(n)\right) \boldsymbol{U}^{-1}
$$

\section{Simulations AND Discussions}

We evaluated the channel estimators using the normalized meansquare error (NMSE) denoted by

$$
\mathrm{NMSE}=\frac{1}{N(K+1) \mathcal{I}} \sum_{\imath=1}^{\mathcal{I}} \frac{\left\|\hat{\tilde{\boldsymbol{H}}}_{\imath}-\tilde{\boldsymbol{H}}\right\|^{2}}{\|\tilde{\boldsymbol{H}}\|^{2}}
$$

where $\mathcal{I}$ is the number of Monte Carlo trials. The reason to use this NMSE definition is that we are not able to obtain the CIR in the subband OFDMA uplink. The estimated channel information $\tilde{\boldsymbol{H}}$ was then used for the channel equalization. The bit error rate (BER), averaged over $\mathcal{I}=500$ trials, was examined for both a single transmit antenna and two transmitantennas. ${ }^{3}$

The setup of OFDMA uplink was given by $M=128$ subcarriers, which was divided into 32 subbands. Each subband consisted of four subcarriers (i.e., $K=3$ ). The information-bearing symbols were drawn from the quadrature phase-shift-keying (QPSK) constellation with the equal probability (uncoded source). The time-frequency pilot/data map is depicted in Fig. 1. Each frame (data burst) consists of 300 blocks. The mobile channel was generated according to the 3GPP Vehicular A specified in [14]. The SNR was defined by the average received symbol energy to noise. The normalized maximum Doppler-frequency $\bar{f}_{\max }$ was considered to be 0.025 or 0.05 , respectively, corresponding to the velocity of $75 \mathrm{~km} / \mathrm{h}$ or $150 \mathrm{~km} / \mathrm{h}$ in the system with 2-GHz central frequency and a block duration of $180 \mu \mathrm{s}$. As mentioned in [5], we focus on terrains entailing only a few reflectors so that the Doppler and multipath parameters can be considered to be deterministic. In the simulations, we use the time-frequency linear interpolation (TFLI) and the least-square (LS) approach (see [2]) for the performance comparison. For the fair comparison, the transmitter sends one training sequence for every four blocks in the LS approach.

1) Experiment 1 (Single Transmit Antenna): The pilot spacing (PS) along the time direction was given by 2 and 4, respectively. The normalized maximum Doppler frequency was assumed to be known at the receiving end. For the case of $\bar{f}_{\max }=0.05$, we set $N=80$ and $Q=$ 4. The channel estimation performance was plotted in Fig. 2. It shows that all curves generally decrease with increasing of the SNR. The LS approach shows the worst performance. The TFLI method has an error floor for the case of SNR $>10 \mathrm{~dB}$ and PS $=4$. This is because of the considerable mismatch between the linear channel model and the time selectivity between two adjacent pilots. The error floor vanishes when the PS is reduced to 2 . The estimation performance is comparable to the HLBI method with the PS of 4. The HLBI method gives the improved estimation performance for the smaller PS $(=2)$, because the number

\footnotetext{
${ }^{3}$ Practically, a mobile terminal is equipped with up to two antennas.
}

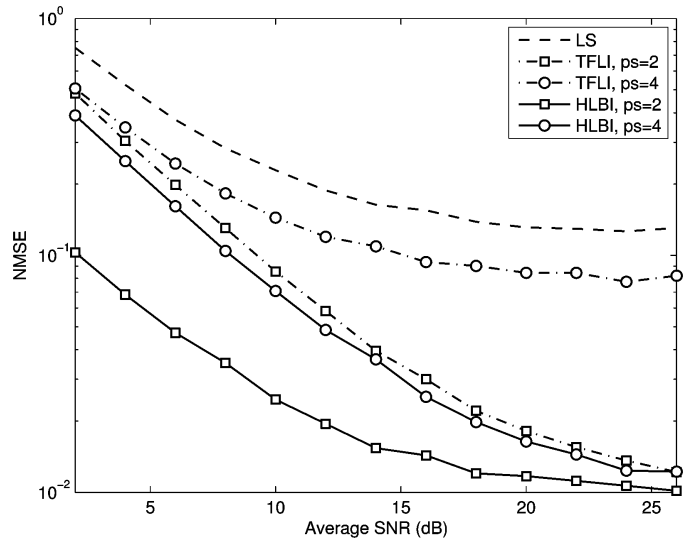

Fig. 2. NMSE versus SNR, single antenna with $\bar{f}_{\max }=0.05$

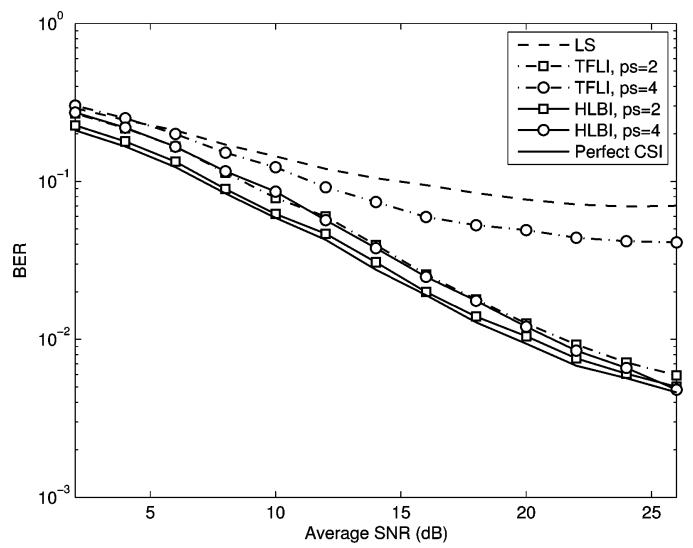

Fig. 3. BER versus SNR, single antenna with $\bar{f}_{\max }=0.05$.

of pilots employed for the channel estimation is doubled. Using the estimated channel information as well as the perfect channel knowledge for the channel equalization, we plot the BER performance in Fig. 3. It is observed that the HLBI method outperforms both the TFLI and LS methods. For the case of PS $=2$, it offers very close performance to that with the perfect channel knowledge. When the number of pilots is reduced to half ( $\mathrm{PS}=4$ ), its performance is still comparable to that with the perfect channel knowledge.

For the case of $\bar{f}_{\max }=0.025$, we set $N=160$ and $Q=4$. Fig. 4 illustrates the channel estimation performance. We can see that both the LS and TFLI methods show the improved performance in comparison to the case of $\bar{f}_{\max }=0.05$. This is because of the reduction of modelling mismatch in the relatively lower time-selectivity scenario. The HLBI method shows the improved performance as well in comparison to the case of $\bar{f}_{\max }=0.05$. The major reason here is due to the increased number of pilots in the channel estimation. The BER performance is then plotted in Fig. 5. We can observe that the HLBI method still demonstrates the best performance. Under the same PS setup, the difference between HLBI and TFLI is around $2 \mathrm{~dB}$ in SNR. Compared to the case with the perfect channel knowledge, the HLBI method offers close performance in the low- and medium-SNR range and only has a $2-\mathrm{dB}$ loss in the high-SNR range $(>20 \mathrm{~dB})$.

2) Experimental 2 (Two Transmit-Antennas): We use Alamouti scheme for data transmission. Then, the equalization can be easily carried out at each receiving antenna. We note that the pilot pattern $[(30),(33)]$ is not applicable in the TFLI method. For the fair comparison, both HLBI and TFLI methods use the pilot pattern (34), which is depicted in Fig. 1(b). Fig. 6 illustrates the channel estimation performance with $\bar{f}_{\max }=0.05$. Observing the NMSE both in single 


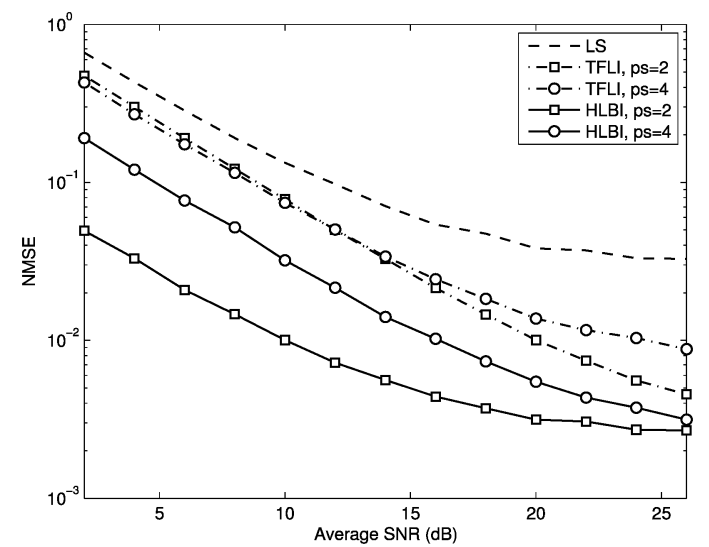

Fig. 4. NMSE versus SNR, single antenna with $\bar{f}_{\max }=0.025$.

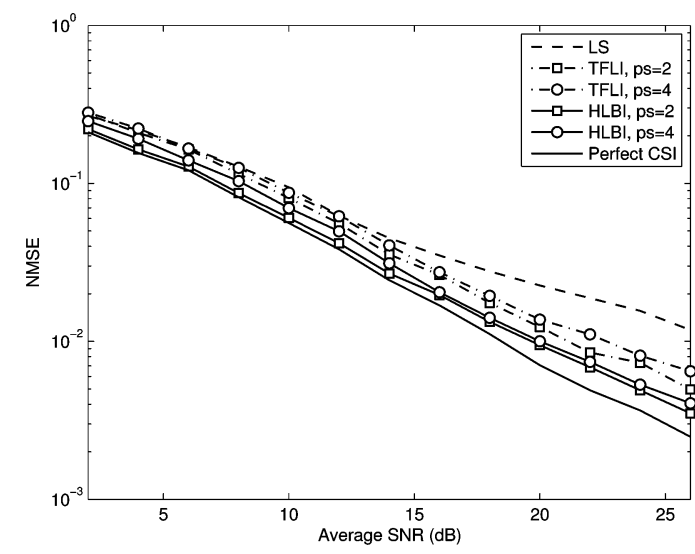

Fig. 5. BER versus SNR, single antenna with $\bar{f}_{\max }=0.025$.

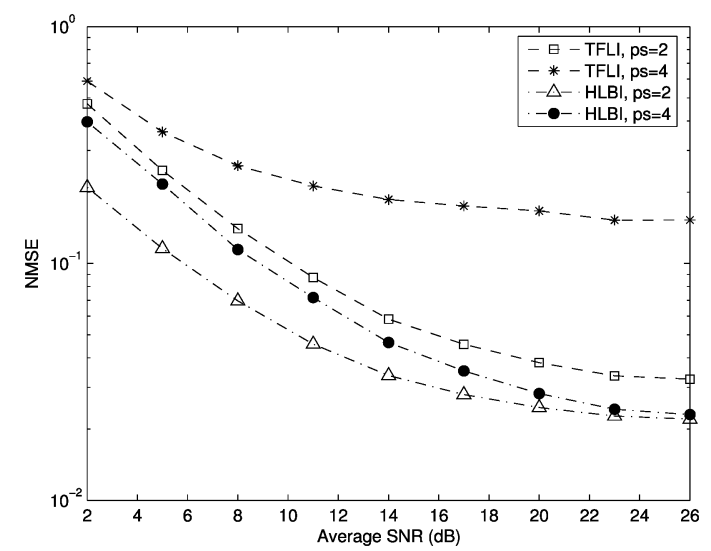

Fig. 6. NMSE versus SNR, two antennas with $\bar{f}_{\max }=0.05$.

transmit-antenna and two transmit-antennas cases, we can find that both HLBI and TFLI methods show the increased estimation error in the later case. This is because employing zero pilots doubles the actual pilot spacing. We can also observe that the major performance difference between HLBI and TFLI is in the low and medium SNR range. This is because HLBI has stronger denoising capability. We then examine the overall system performance and plot the BER in Fig. 7. It is shown that Alamouti scheme is sensitive to $\bar{f}_{\max }$ as well as PS (see the case with the perfect channel knowledge). This is because the space-time code was originally proposed for the slowly time-varying channel. The HLBI method shows almost the same performance as the case with the perfect channel knowledge.

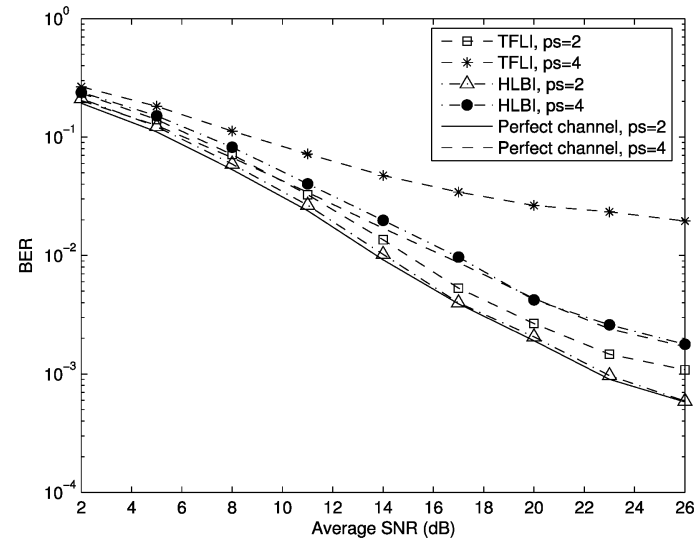

Fig. 7. BER versus SNR, two antennas with $\bar{f}_{\max }=0.05$.

\section{CONCLUSION}

In this paper, we have presented an HLBI-based channel estimation method for the subband OFDMA uplink. The proposed method was based on the hybrid of linear and BEM channel model of the partial channel information. The channel estimation performance and two-dimensional pilot pattern have been analytically investigated in both single and multiple transmit antennas. Simulation results were provided to show the NMSE and BER performance. It has been shown that the HLBI method has stronger denoising capability than the TFLI method. Its estimation performance would not been considerably affected by the Doppler frequency when the parameters for the HLBI method are set properly. Its overall system performance is comparable to the case with the perfect channel knowledge. The proposed method is particularly suitable to the mobile environment where multipath propagation is caused by a few dominant reflectors.

\section{ACKNOWLEDGMENT}

The authors would like to thank the associate editor Prof. P. Loubaton and the reviewers for the efficient review process and constructive comments.

\section{REFERENCES}

[1] R. Negi and J. Cioffi, "Pilot tone selection for channel estimation in a mobile OFDM system," IEEE Trans. Consum. Electron., vol. 44, pp. 1122-1128, Aug. 1998.

[2] O. Edfors, M. Sandell, J.-J. van de Beek, S. K. Wilson, and P. O. Borjesson, "OFDM channel estimation by singular value decomposition," IEEE Trans. Commun., vol. 46, pp. 931-939, Jul. 1998.

[3] Y. Li, L. J. Cimini, Jr, and N. Scollenberger, "Robust channel estimation for OFDM systems with rapid dispersive fading channels," IEEE Trans. Commun., vol. 46, pp. 902-915, Jul. 1998.

[4] D. K. Borah and B. T. Hart, "Frequency selective fading channel estimation with a polynomial time-varying channel model," IEEE Trans. Commun., vol. 48, pp. 862-873, Jun. 1999.

[5] G. B. Giannakis and C. Tepedelenlioglu, "Basis expansion models and diversity techniques for blind identification and equalization of timevarying channels," Proc. IEEE, vol. 86, pp. 1969-1986, Oct. 1998.

[6] X. Ma and G. B. Giannakis, "Maximum-diversity transmissions over doubly selective wireless channels," IEEE Trans. Info. Theory, vol. 49, pp. 1832-1840, Jul. 2003.

[7] X. Ma, G. B. Giannakis, and S. Ohno, "Optimal training for block transmissions over doubly selective wireless fading channels," IEEE Trans. Signal Process., vol. 51, no. 5, pp. 1351-1366, May 2003.

[8] S. Ohno and G.B. Giannakis, "Optimal training and redundant precoding for block transmissions with application to wireless OFDM," IEEE Trans. Commun., vol. 50, pp. 2113-2123, Dec. 2002.

[9] M. Siala, "Maximum a posteriori fast fading channel estimation based exclusively on pilot symbols," Ann. Telecommun., vol. 56, no. 9-10, Sep./Oct. 2001. 
[10] — " "Maximum a posteriori semi-blind channel estimation for OFDM systems," Ann. Telecommun., vol. 57, no. 9-10, Sep./Oct. 2002.

[11] T. Zemen and C. F. Mecklenbräuker, "Time-variant channel estimation using discrete prolate spheroidal sequences," IEEE Trans. Signal Process., vol. 53, no. 9, pp. 3597-3607, Sep. 2005.

[12] Z. Wang and G. B. Giannakis, "Wireless multicarrier communications: Where Fourier meets Shannon," IEEE Signal Process. Mag., vol. 17, pp. 29-48, May 2000.

[13] S. M. Alamouti, "A simple transmit diversity technique for wireless communications," IEEE J. Sel. Areas Commun., vol. 16, pp. 1451-1458, Oct. 1998.

[14] "3rd Generation Partnership Project; Technical Specification Group Radio Access Network; Spatial Channel Model for Multiple Input Multiple Output (MIMO) Simulations (Release 6)," Document 3GPP TR 25.996 v6.1.0, 2003

\section{Regularized Channel Diagonalization for Multiuser MIMO Downlink Using a Modified MMSE Criterion}

\author{
Jingon Joung, Student Member, IEEE, and \\ Yong H. Lee, Senior Member, IEEE
}

\begin{abstract}
We propose a regularized channel diagonalization method for a joint transmit-receive linear optimization in the downlink of a multiuser multiple-input multiple-output (MIMO) communication system. This method is based on the use of a modified minimum mean-square error (MMSE) criterion, which employs a weighted information symbol vector for the target and signal scaling. The weights for the target are the equivalent channel gain resulting from a zero-forcing (ZF)-based MIMO channel diagonalization. A joint iterative algorithm for minimizing the mean-square error (MSE) under a total transmit power constraint is derived, and its convergence is proved. The signal-to-interference-plus-noise ratio (SINR) is analyzed and the sum rates evaluated in a computer simulation. The results demonstrate that the proposed method outperforms the existing ZF- and MMSE-based methods.
\end{abstract}

Index Terms-Downlink, minimum mean-square error (MMSE), multiuser multiple-input multiple-output (MIMO), spatial multiplexing, zeroforcing (ZF).

\section{INTRODUCTION}

Spatial multiplexing for multiple-input multiple-output (MIMO) radio systems, employing multiple transmit and receive antennas, has been recognized as an effective way to improve the spectral efficiency of wireless links [1]-[3]. This is realized by transmitting multiple data substreams in parallel and performing some transmit-receive processing which assists data recovery. In a multiuser MIMO downlink, where the base station communicates simultaneously with multiple users, the data substreams are generated by combining the signals of different users (Fig. 1). To mitigate the cochannel interference (CCI) caused by the spatial multiplexing in a multiuser downlink, a channel inversion and its modifications [4]-[7] have been introduced in the

Manuscript received May 11, 2005; revised June 23, 2006. The associate editor coordinating the review of this manuscript and approving it for publication was Dr. Timothy N. Davidson. This work was supported in part by the University Information Technology Research Center Program of the government of Korea.

The authors are with the School of Electrical Engineering and Computer Science, Korea Advanced Institute of Science and Technology, Daejeon 305-701, Korea (e-mail: yohlee@ee.kaist.ac.kr).

Digital Object Identifier 10.1109/TSP.2006.890825 form of transmit processing algorithms. These are mainly useful for systems with single-antenna receivers. For systems with multiple receiver antennas, coordinated transmit-receive processors have been developed based on a joint-channel diagonalization ${ }^{1}$ [8]-[11] and a minimum mean-square error (MMSE) criterion [12]-[17]. The channel diagonalization methods are zero-forcing (ZF) algorithms that attempt to eliminate the CCI, while ignoring noise. On the other hand, the MMSE algorithms control the degree of CCI suppression depending on the signal-to-noise ratio (SNR). Accordingly, the MMSE schemes outperform the channel diagonalization methods in low SNR environment [15].

In this paper, an alternative MMSE scheme is proposed that modifies the total-MMSE (T-MMSE) algorithm in [15]. It is observed that the T-MMSE performs a regularized channel inversion and acts like a channel inverter in high SNR environment. Consequently, the sum rate performance of the T-MMSE tends to become worse than that of the channel diagonalization methods as the SNR increases. To avoid this performance degradation, the MMSE criterion is modified so that the resulting MMSE scheme performs regularized channel diagonalization. Specifically, the MMSE criterion employs a target vector which is given by a weighted information vector, where the weights are the equivalent channel gain resulting from a joint-channel diagonalization, and signal scaling. Due to the use of the weights, the proposed approach can assign more power to stronger subchannels. The signal scaling enables us to derive a closed-form expression for the Lagrange multiplier, which is employed to consider a transmit power constraint [18]. An iterative algorithm for minimizing the MSE under a total transmit power constraint is derived, and its characteristics are analyzed. It will be shown that the proposed algorithm, termed the modified T-MMSE (MT-MMSE), can outperform the existing methods irrespective of the SNR.

The organization of this paper is as follows. Section II describes the multiuser MIMO system model. The proposed method is then derived and analyzed in Section III. Section IV presents computer simulation results to demonstrate the advantage of the proposed processors. Finally, Section V presents the conclusion.

\section{Multiuser MiMO System Model}

The system configuration of a multiuser MIMO downlink with $K$ users, $N_{T}$ transmit antennas, and $N_{R, k}$ receive antennas, $k \in\{1,2, \ldots, K\}$, is shown in Fig. 1. The MIMO channel is represented as $\mathbf{H}_{k} \in \mathbb{C}^{N_{R, k} \times N_{T}}$, where the entries are independently identically distributed (i.i.d.) zero-mean complex Gaussian random variables with a unit variance. The $(m, n)$ th entry represents the complex gain from the $n$th transmit antenna to the $m$ th receive antenna. It is assumed that all $\left\{\mathbf{H}_{k}\right\}$ are known at the transmitter, while the $k$ th receiver only knows its own MIMO channel. This assumption indicates that the multiuser interference should be suppressed at the transmitter via preprocessing. The spatial multiplexing is performed by forming a vector signal $\mathbf{x}_{k}$ with $L_{k}$ symbols, $\mathbf{x}_{k} \in \mathbb{C}^{L_{k} \times 1}$, preprocessing each vector by $\mathbf{T}_{k}$, where $\mathbf{T}_{k} \in \mathbb{C}^{N_{T} \times L_{k}}$ is the transmit-processing matrix, and combining the preprocessed vectors from the users to yield $\sum_{k=1}^{K} \mathbf{T}_{k} \mathbf{x}_{k}$. Following this, the elements of $\sum_{k=1}^{K} \mathbf{T}_{k} \mathbf{x}_{k}$ are transmitted through different antennas. At the $k$ th receiver, the received signal $\mathbf{H}_{k} \sum_{j=1}^{K} \mathbf{T}_{j} \mathbf{x}_{j}+\mathbf{n}_{k}$, where $\mathbf{n}_{k} \in \mathbb{C}^{N_{R, k} \times 1}$ is a

${ }^{1}$ After the channel diagonalization, the equivalent channel gain matrix, which describes the cascade of transmit-receive processing and the physical channel, becomes a diagonal matrix. Channel inversion is a special case of channel diagonalization in which the equivalent channel gain is given by a normalized identity matrix. 